\title{
Isolation, Characterization and Screening for Anticancer and Antimicrobial Properties of the Crude Extract from Genus Neosartorya
}

\author{
Supattra Poeaim*, Kongluekai Tongkantom, Prapaiporn Jabamrung, On Bo-kaew, Mayamor Soytong \\ Department of Biology, Faculty of Science, King Mongkut's Institute of Technology Ladkrabang, Thailand
}

Copyright $\mathrm{C} 2016$ by authors, all rights reserved. Authors agree that this article remains permanently open access under the terms of the Creative Commons Attribution License 4.0 International License

\begin{abstract}
The genus Neosartorya, the sexual states of Aspergillus, belongs to the Eurotiales which have been reported for compounds with bioactivities such as antimicrobial, antimalarial and cytotoxicity. The objective of this research was to isolate, identify and screening for anticancer and antimicrobial properties of the ethyl acetate crude extract from Neosartorya. Neosartorya sp. was isolated from soil in Chiang Mai province, Thailand by the soil plate technique. The samples were identified base on morphology and DNA sequencing: Internal transcribed spacer (ITS), $\beta$-tubulin and calmodulin as Neosartorya hiratsukae and Neosartorya pseudofischeri. For in vitro cytotoxicity activity by MTT assay using eight cell lines (MCF-7, P388, HeLa, Vero, L929, KB, HT-29 and HepG2). The crude ethyl acetate extract of $N$. hiratsukae and $N$. pseudofischeri showed the highest potency against the L929 cell line with the $50 \%$ inhibitory concentration $\left(\mathrm{IC}_{50}\right)$ value of 144.31 and $267.73 \mu \mathrm{g} / \mathrm{ml}$, respectively. The antimicrobial properties of crude extract were evaluated using disc diffusion method against five bacteria strain: Bacillus subtilis, Staphylococcus aureus, Micrococcus luteus, Escherichia coli and Pseudomonas aeruginosa. The extract was well inhibited the growth of gram positive pathogenic bacteria: B. subtilis, S. aureus and M. luteus. The results indicate its possible potential with the production of other bioactive agents for future anticancer and antibiotic drug development.
\end{abstract}

Keywords Antimicrobial, Cytotoxicity, Neosartorya hiratsukae, Neosartorya pseudofischeri

\section{Introduction}

The genus Neosartorya belongs to Trichocomaceae family under the order Eurotiales. These fungi are perfect state (teleomorphic stage) of Aspergillus. Some species of the genus Neosartorya have been reported for compounds with bioactivities such as antibacterial and cytotoxicity. For example, three new compounds isolated from the Neosartorya pseudofischeri inhibited the growth of six human cancer cell lines that a quinazolinone displayed cytostatic effects in human U373 glioblastoma and A549 non-small cell lung cancer apoptosis-resistant cells with marked inhibition of mitotic rates [1]. New compounds, a pyrroloindole and fischerindoline, inhibited the growth of six human and mouse cancer cell lines [2]. Including, a new meroterpenoid: tatenoic acid was isolated from the fungus $N$. tatenoi exhibited cytotoxicity against human small cell lung cancer (NCI-H187) and human epidermoid carcinoma in the mouth (KB) [3]. Additionally, the crude mycelial extracted from $N$. spinosa were reported as antibacterial [4]. The screening for bioactive compounds from the Neosartorya is challenging. Therefore, this research focuses on isolation and identification of Neosartorya from the forest soil in Thailand and screening for anticancer and antimicrobial properties.

\section{Materials and Methods}

\subsection{Fungus Isolation and Morphological Characterization}

The genus Neosartorya were isolated from soil collected at Doi Suthep and Doi Inthanon mountains (Chiang Mai, Thailand). The soil samples were cultured on glucose ammonium nitrate agar (GANA) by soil plate technique [5]. Then, single colony of Neosartorya was transferred to PDA for purification. The fungi were grown on PDA about 2 weeks at $25^{\circ} \mathrm{C}$ for morphological characterization. The identification was done by observation of colony and mycelia morphology, cleistothecia, asci and ascospore using light microscopy and scanning electron microscopy [5-6].

\subsection{DNA Extraction, Amplification and Sequencing}


Fungal genomic DNA was obtained from 10-15 days old culture mycelia of the potato dextrose broth medium (PDB) and genomic DNA extracted using the standard $\mathrm{CTAB}$ method. The ITS ribosomal DNA regions were amplified by PCR using the universal primers, ITS1 and ITS4 [7]. The $\beta$-tubulin gene was amplified using the Bt2a and Bt2b [8] and the calmodulin gene was amplified using the CF1L and CF4 primers [9]. The PCR products were purified with PCR purified kit (Vivantis, USA). Sequencing was performed at First Base Laboratories, Malaysia. The obtained nucleotide sequences were searched through BLASTN at GenBank database (http://www.ncbi.nlm.nih.gov/BLAST).

\subsection{Fungal Culture and Extraction}

The mycelium was taken by vertically cutting $6 \mathrm{~mm}$ diameter plugs using a cork borer. The plugs were transferred to $250 \mathrm{ml}$ bottles containing $10 \mathrm{ml}$ of PDB and incubated at room temperature for 1 month. The culture was harvested by filtration through several layers of gauze and mycelia were collected and dried at $45^{\circ} \mathrm{C}$ for 3 days. The powdered mycelia were extracted with ethyl acetate on a rotary shaker (120 rpm) for 3 days at room temperature and repeated for 3 times. The supernatants were filtered through a Whatman filter paper No. 1 and concentrated using a rotary evaporator at $40^{\circ} \mathrm{C}$. The crude extracts were kept in dark bottles under refrigerated conditions until the time of experiment.

\subsection{Determination of Anticancer Activity}

The HT-29 (Human colon adenocarcinoma), MCF-7 (Human breast carcinoma), P388 (Murine leukemia), L929 (Murine fibroblasts) and Vero (African green monkey kidney) cell lines were a kind gift from Dr. Porntipa Picha, Research Division, National Cancer Institute, Bangkok. The KB (Human mouth epidermal carcinoma), HeLa (Human cervical carcinoma) and HepG2 (Human hepatocellular carcinoma) cell lines were obtained from Scientific Instruments Centre, Faculty of Science, King Mongkut's Institute of Technology Ladkrabang, Bangkok. The cells were maintained in RPMI 1640 medium with $10 \%(\mathrm{v} / \mathrm{v})$ fetal bovine serum (FBS) and $0.05 \mu \mathrm{g} / \mathrm{ml}$ gentamicin in $5 \% \mathrm{CO}_{2}$ humidified incubator at $37^{\circ} \mathrm{C}$. The MTT assay proposed by Mosmann [10] was modified and used to determine antiproliferative activity of extracts. Briefly, exponentially growing cells were seeded onto a 96-well plate and allowed to attach for 24 hours before treatment. The crude extracts were dissolved in dimethyl sulfoxide (DMSO) and sterilized by filtration through $0.22 \mu \mathrm{m}$ filter. Cells were treated with $100 \mu \mathrm{l}$ of those crude extracts within a range of final concentrations from 125 to $2000 \mu \mathrm{g} / \mathrm{ml}$ for 20 hours. The fifty microliters of MTT solution $(2 \mathrm{mg} / \mathrm{ml}$ in phosphate buffer saline) were added to each well and further incubated for 4 hours at $37^{\circ} \mathrm{C}$. Twenty five microgram per milliliter of Mitomycin C (MMC) and 1\% DMSO final concentration were added as a positive and negative control, respectively.
All the exposure conditions were tested in triplicate and observed for cell morphological analysis. After the reaction, the supernatant were removed and replaced with DMSO: ethanol $(1: 1 \mathrm{v} / \mathrm{v})$ to solubilized formazan crystals. The absorbance was measured at $570 \mathrm{~nm}$ using a microplate reader (Anthos MultiRead 400, Biochrom, UK). The percentage of inhibition of cell growth was calculated and the $50 \%$ inhibitory concentration $\left(\mathrm{IC}_{50}\right)$ was estimated using GraphPad Prism 5 software.

\subsection{Determination of Antimicrobial Activity}

The extracts were tested using agar disc diffusion described by Ansari et al. [11]. Five microbial strains: Bacillus subtilis, ATCC6633, Micrococcus luteus ATCC9341 and Staphylococcus aureus TISTR1466 and two gram negative bacteria (Escherichia coli ATCC25922 and Pseudomonas aeruginosa ATCC27853 were cultured in Mueller-Hinton Broth (MHB) and incubated at $37^{\circ} \mathrm{C}$ for $4-5$ hours. The optical density of cells was adjusted to $0.5-0.8$ at $600 \mathrm{~nm}$. Each bacterial suspension was swab over the surface of Mueller Hinton Agar (MHA) with a sterile cotton swab. The dried crude extract was dissolved in a methanol. Various concentrations of extracts $(0.20,0.5,1$ and $2 \mu \mathrm{g} / \mathrm{disc})$ were loaded onto each the $6 \mathrm{~mm}$ sterile paper disc, allowed to dry (about 30 min incubation) then was placed onto inoculated plates. Twenty microliters of methanol and $100 \mu \mathrm{g} / \mathrm{ml}$ gentamicin were used as negative and positive control, respectively. Incubated at $37^{\circ} \mathrm{C}$ for 24 hours, the diameters of the inhibition zone were measured.

\section{Results and Discussion}

\subsection{Isolation, Morphological and DNA Sequencing Characterization}

The morphological features were observed as microscopic characters which showed white to creamy colony and cleistothecia, ascospores are globose to broadly ellipsoidal with equatorial furrow and ridges identified as Neosartorya. EU06 had a creamy-white colony, cleistothecia are globose $(90.7-263.05 \times 91.98-276.76 \mu \mathrm{m})$, ascus is globose (9.86-13.63 $\times 8.63-13.9 \mu \mathrm{m})$ and ascospores are broadly ellipsoidal $(3.86-6.81 \times 3.70-6.48 \mu \mathrm{m})$ with two equatorial crests (Figure 1). The other (EU13) had a white colony, cleistothecia are globose (274.44-506.11 x301.43-526.58 $\mu \mathrm{m})$, ascus is subglobose $(10.70-12.03 \times 11.23-12.08 \mu \mathrm{m})$ and ascospores are subglobose $(3.54-5.53 \times 4.30-5.59 \mu \mathrm{m})$ with narrow equatorial furrow (Figure 2). These indicate that identification of Neosartorya sp. not easy based on morphology. Therefore, we further analyzed DNA sequence of the three regions and combined the data with morphological characteristic in order to confirm species. The nucleotide sequences were compared with BLASTn in NCBI Genbank. The three region were amplified that related to the 
two species. For EU06, the fragments for ITS, $\beta$-tubulin and calmodulin were 606,545 and $735 \mathrm{bp}$, respectively identified as $N$. hiratsukae. For EU13, the fragments for ITS, $\beta$-tubulin and calmodulin were 588, 553 and $764 \mathrm{bp}$, respectively identified as $N$. pseudofischeri. The dendrogram of calmodulin gene was demonstated representative of these fungi (Figure 3). Our result was supported with findings of Sanmanoch et al. [4] who reported the nucleotide sequences from the ITS region is useful and suitable for Neosartorya identification. However, identification of Neosartorya should be using both molecular and morphological techniques [12].

\subsection{Anticancer Activity}

Antiproliferative activity of ethyl acetate extract of $N$. hiratsukae and $N$. pseudofischeri were determined using the MTT assay in eight cell lines (HT-29, MCF-7, P388, L929, Vero, KB, HeLa and HepG2) at concentration ranging from 125 to $2000 \mu \mathrm{g} / \mathrm{ml}$. Pretreatment with MTT solution, the cells treated with extract were irregular and round shape when observed under inverted phase contrast microscope. MMC used for positive control induced differences cytotoxicity in cell lines (cytotoxicity $>50 \%$ : data not shown). The crude extract of $N$. hiratsukae exhibited antiproliferative activity against the top three cell lines; L929, Vero and HeLa, respectively. The ethyl acetate extract of $N$. pseudofischeri shown against the top three cell lines; L929, HeLa and HT-29, respectively. The results also show that L929 cell line was the most sensitive to the $N$. hiratsukae and $N$. pseudofischeri extracts with $\mathrm{IC}_{50}$ values are 144.30 and $267.73 \mu \mathrm{g} / \mathrm{ml}$, respectively (Table 1). Our findings are consistent with the some species of the genus Neosartorya such as $N$. pseudofischeri, $N$. tatenoi and $N$. spinosa have been reported for compounds which potent cytotoxicity against the mammalians and insect cell lines $[1-4,13]$.

\subsection{Antimicrobial Activity}

The crude ethyl acetate extract of $N$. hiratsukae and $N$. pseudofischeri showed antimicrobial activity against three of five tested human pathogenic bacteria. No inhibitory activity against $E$. coli and $P$. aeruginosa was observed. Crude extracts from Neosartorya showed well inhibited the growth of gram positive bacteria (B. subtilis, S. aureus and M. luteus) (Table 2). Our result was supported with findings of Sanmanoch et al. [4] who reported to the antibacterial activities of crude ethyl acetate extract from $N$. spinosa KKU1NK1 most strongly inhibited the growth of gram positive pathogenic bacteria (S. aureus, S. saprophyticus, $S$. pneumonia and B. subtilis).

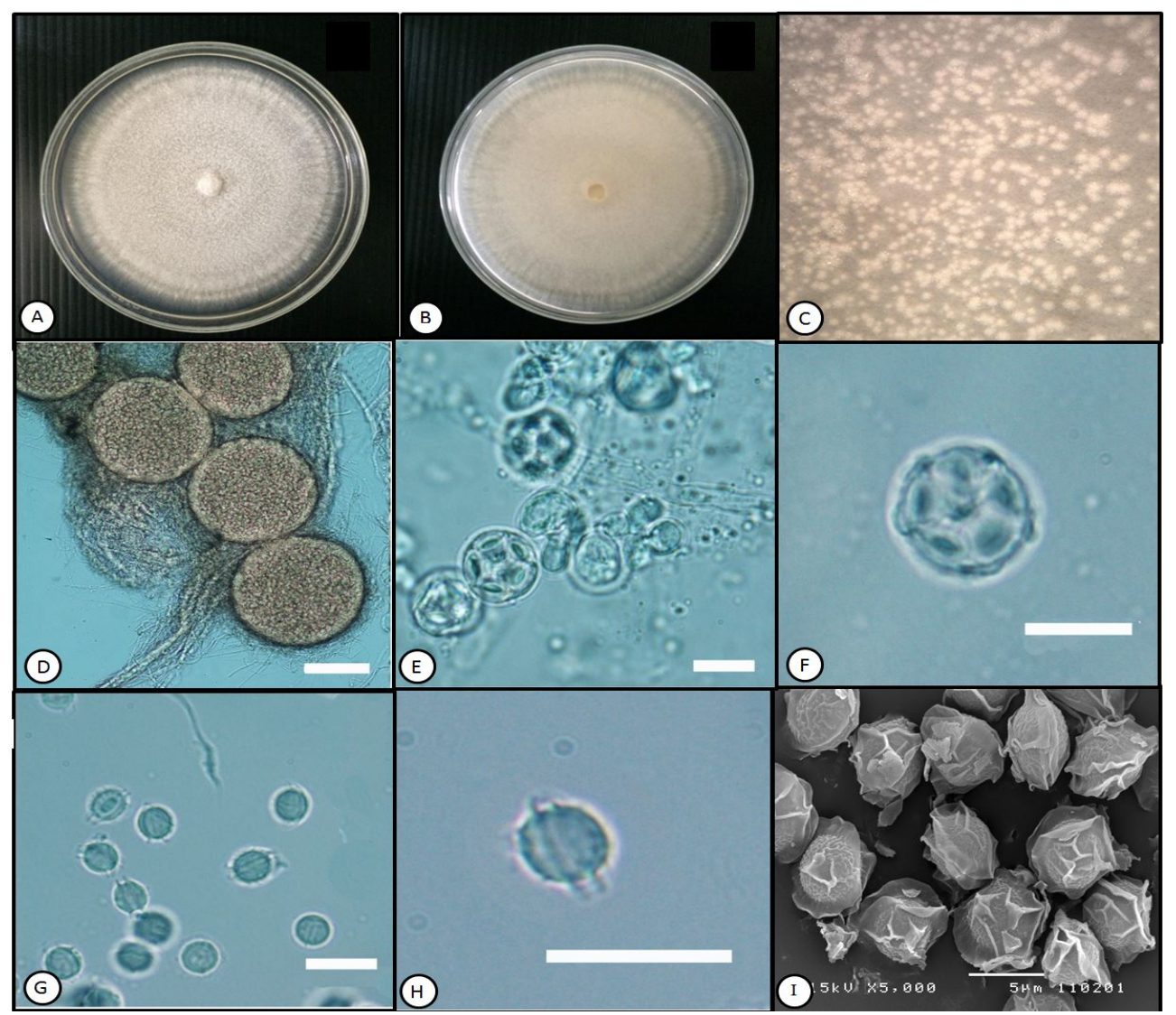

Figure 1. Morphological features of Neosartorya hiratsukae, A-B: Colony grown on PDA for 15 day at $25^{\circ} \mathrm{C}, \mathrm{C}-\mathrm{D}$ : Cleistothecia, E: Asci exposed from cleistothecium, F: Ascus, G-H: Ascospores observed under light microscope and I: Ascospores as seen by SEM. Bars, $100 \mu \mathrm{m}$ (D) and $10 \mu \mathrm{m}$ (E-H). 


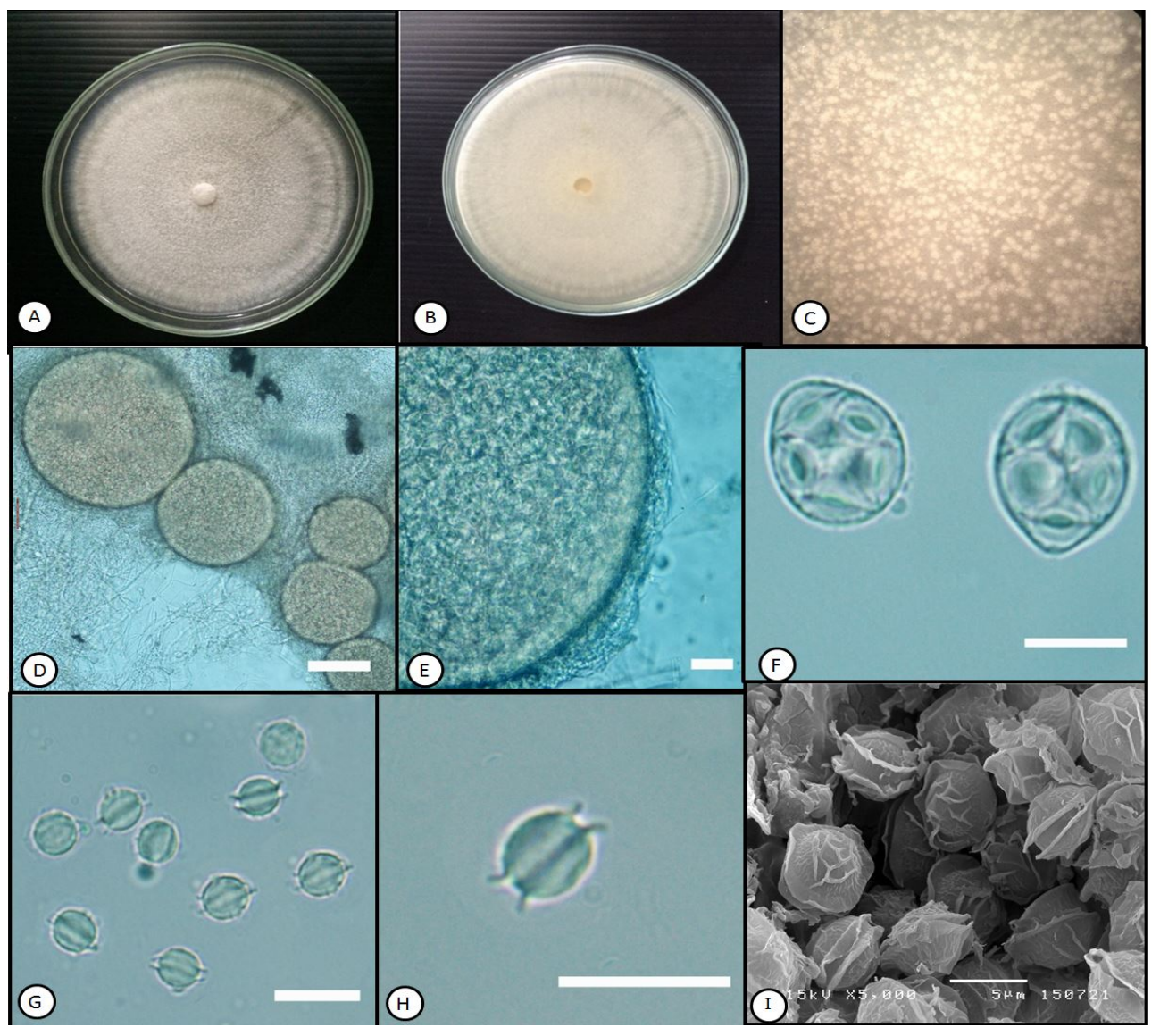

Figure 2. Morphological features of Neosartorya pseudofischeri, A-B: Colony grown on PDA for 15 day at $25^{\circ} \mathrm{C}, \mathrm{C}-\mathrm{D}$ : Cleistothecia, E: Cleistothecium, F: Asci, G-H: Ascospores observed under light microscope and I: Ascospores as seen by SEM. Bars, $100 \mu \mathrm{m}$ (D), $20 \mu \mathrm{m}$ (E) and $10 \mu \mathrm{m}$ (F-H).

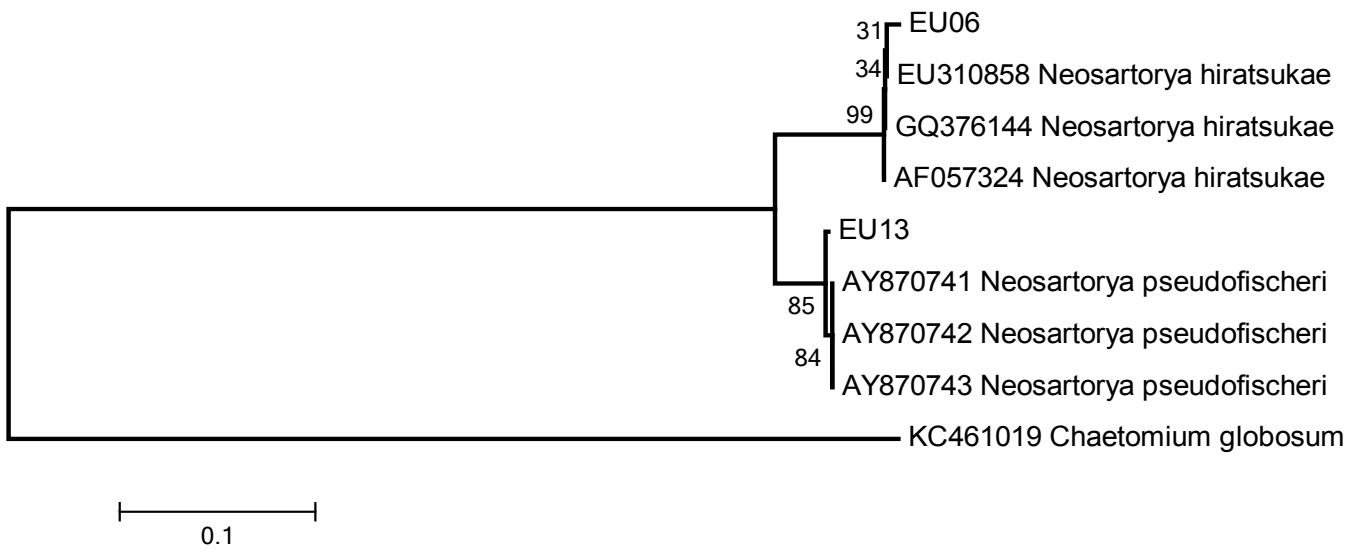

Figure 3. The dendrogram of Neosartorya hiratsukae and Neosartorya pseudofischeri based on calmodulin gene using Neighbor-joining, bootstrap $=1000$

Table 1. Cytotoxic activity of ethyl acetate extract of the N. hiratsukae and N. pseudofischeri

\begin{tabular}{|c|c|c|c|}
\hline \multirow{2}{*}{ Type } & \multirow{2}{*}{ Cell lines } & \multicolumn{2}{|c|}{$50 \%$ inhibitory concentration: $\mathrm{IC}_{50}(\mu \mathrm{g} / \mathrm{ml})$} \\
\cline { 2 - 4 } & P388 & N. hiratsukae & N. pseudofischeri \\
\hline \multirow{4}{*}{ Cancer cell lines } & MCF-7 & $1,464.45$ & 714.21 \\
\cline { 2 - 4 } & HT-29 & $1,368.31$ & $1,673.19$ \\
\cline { 2 - 4 } & HepG2 & $1,235.14$ & 702.23 \\
\cline { 2 - 4 } & HeLa & $1,783.78$ & $>2,000.00$ \\
\cline { 2 - 4 } & KB & 913.07 & 461.11 \\
\hline \multirow{2}{*}{ Normal cell lines } & Vero & $1,584.39$ & $1,581.27$ \\
\cline { 2 - 4 } & L929 & 687.79 & $1,038.13$ \\
\hline
\end{tabular}


Table 2. Antibacterial activity of crude ethyl acetate extracts from N. hiratsukae and N. pseudofischeri using paper disc diffusion method

\begin{tabular}{|c|c|c|c|c|}
\hline \multirow{3}{*}{ Crude extracts } & \multirow{2}{*}{ Concentration $(\mu \mathrm{g} / \mathrm{disc})$} & \multicolumn{3}{|c|}{${\text { Zone of inhibition }(\mathrm{mm})^{*}}^{*}$} \\
\cline { 2 - 5 } & 2 & B. subtilis & M. luteus & S. aureus \\
\hline \multirow{3}{*}{ N. hiratsukae } & 1 & $10.05 \pm 0.02$ & $13.94 \pm 0.44$ & $8.99 \pm 1.26$ \\
\cline { 2 - 5 } & 0.5 & $9.82 \pm 0.09$ & $11.98 \pm 1.14$ & $8.20 \pm 0.98$ \\
\cline { 2 - 5 } & 0.2 & $8.17 \pm 0.39$ & $10.54 \pm 0.80$ & $6.97 \pm 0.30$ \\
\hline \multirow{3}{*}{ N. pseudofischeri } & 2 & $7.83 \pm 0.12$ & $9.08 \pm 0.85$ & $6.63 \pm 0.30$ \\
\cline { 2 - 5 } & 1 & $11.74 \pm 3.70$ & $9.32 \pm 0.63$ & $10.32 \pm 0.63$ \\
\cline { 2 - 5 } & 0.5 & $9.61 \pm 2.80$ & $7.98 \pm 0.16$ & $8.91 \pm 0.16$ \\
\cline { 2 - 5 } & 0.2 & $9.10 \pm 2.70$ & $7.18 \pm 0.24$ & $7.57 \pm 0.24$ \\
\hline Gentamicin & 2 & $7.50 \pm 0.50$ & $6.98 \pm 0.29$ & $6.53 \pm 0.68$ \\
\hline
\end{tabular}

*Values represent mean $( \pm \mathrm{SE})$ of three experiments each set up in triplicate

\section{Conclusions}

The genus Neosartorya were isolated from forest soil collected at Doi Suthep and Doi Inthanon mountains, Thailand. Identification of Neosartorya species are not necessarily based on morphology, it should be used both morphological characterization and molecular methods. Screening for its bioactive compound, the crude ethyl acetate extract of the $N$. hiratsukae and $N$. pseudofischeri were analyzed to eight different mammalian cell lines which showed potent cytotoxic effect in some cell lines. Additionally, the crude extracts inhibited the growth of gram positive bacteria. Our findings provide further evidence that the fungus Neosartorya has the tremendous potential of biosynthesis. Therefore, our attempts to isolate Neosartorya from different place or sources and screening for their bioactivities in order to development as an anticancer and antibacterial drug.

\section{Acknowledgements}

We would like to thank Assoc. Prof. Dr. Kasem Soytong for his comments and criticizes our manuscript and thanks anonymous reviewers for their helpful comments on the manuscript.

\section{REFERENCES}

[1] A. Eamvijarn, A. Kijjoa, C. Bruyère, V. Mathieu, L. Manoch, F. Lefranc, A. Silva, R. Kiss, W. Herz. Secondary metabolites from a culture of the fungus Neosartorya pseudofischeri and their in vitro cytostatic activity in human cancer cells, Planta Medical, Vol.78, No.16, 1767-1776, 2012.

[2] M. Masi, A. Andolfi, V. Mathieu, A. Boari, A. Cimmino, L.M.Y. Banuls, M. Vurro, A. Kornienko, R. Kiss, A. Evidente. Fischerindoline, a Pyrroloindole Sesquiterpenoid isolated from Neosartorya pseudofischeri with in vitro growth inhibitory activity in human cancer cell lines, Tetrahedron, Vol.69, No.35, 7466-7470, 2013.
[3] T. Yim, K. Kanokmedhakul, S. Kanokmedhakul, W. Sanmanoch, S. Boonlue. A new meroterpenoid tatenoic acid from the fungus Neosartorya tatenoi KKU-2NK23, Natural Product Research: Formerly Natural Product Letters, Vol.28, No.21, 1847-1852, 2014.

[4] W. Sanmanoch, W. Mongkolthanaruk, S. Kanokmedhakul, T. Aimi, S. Boonlue. Isolation of Ascomycetous fungi, Neosartorya spp. and screening for its antibacterial metabolites, Journal of Life Sciences and Technologies, Vol.1, No.3, 180-183, 2013.

[5] K, Soytong. Antagonism of Chaetomium cupreum to Pyricularia oryzae, Journal of Plant Protection in the Tropics, Vol. 9, No.3, 17-23, 1992.

[6] K.H. Domsch, W. Gams, T.H. Anderson. Compendium of soil fungi, IHW-Verlag, Eching, 1993.

[7] T.J. White, T. Bruns, S. Lee, J. Taylor. PCR protocols: a guide to methods and applications, (M.A. Innis, D.H. Gelfand, J.J. Sninsky, T.J. White, eds.), Academic Press, Orlando, Florida, 1990.

[8] V. Hubka, M. Kolarik. $\beta$-tubulin paralogue tubC is frequently misidentified as the benA gene in Aspergillus section Nigri taxonomy: primer specificity testing and taxonomic consequences, Persoonia, Vol.29, 1-10, 2012.

[9] S.W. Peterson. Phylogenetic analysis of Aspergillus species using DNA sequences from four loci, Mycologia, Vol.100, No.2, 205-226, 2008.

[10] T. Mosmann. Rapid colorimetric assay for cellular growth and survival: Application to proliferation and cytotoxicity assays, Journal of Immunological Method, Vol.65, No.1-2, 55-63, 1983.

[11] M.A. Ansari, L. Tirry, M. Moens. Antagonism between entomopathogenic fungi and bacterial symbions of entomopathogenic nematodes, BioControl, Vol.50, No.3, 465-475, 2005.

[12] S.B. Hong, H.S. Cho, H.D. Shin, J.C. Frisvad, R.A. Samson. Novel Neosartorya species isolated from soil in Korea. International Journal of Systematic and Evolutionary Microbiology, Vol.56, No.2, 477-486, 2006.

[13] W.J. Lan, S.J. Fu, M.Y. Xu, W.L. Liang, C.K. Lam, G.H. Zhong, J. Xu, D.P. Yang, H.J. Li. Five new cytotoxic metabolites from the marine fungus Neosartorya pseudofischeri Marine Drugs, Vol.14, No.1, 13 pp, 2016. 